Abstract S106 Table 1 Odds ratios for various factors resulting in LOS > median. Numbers on $y$ axis correspond to first column in below

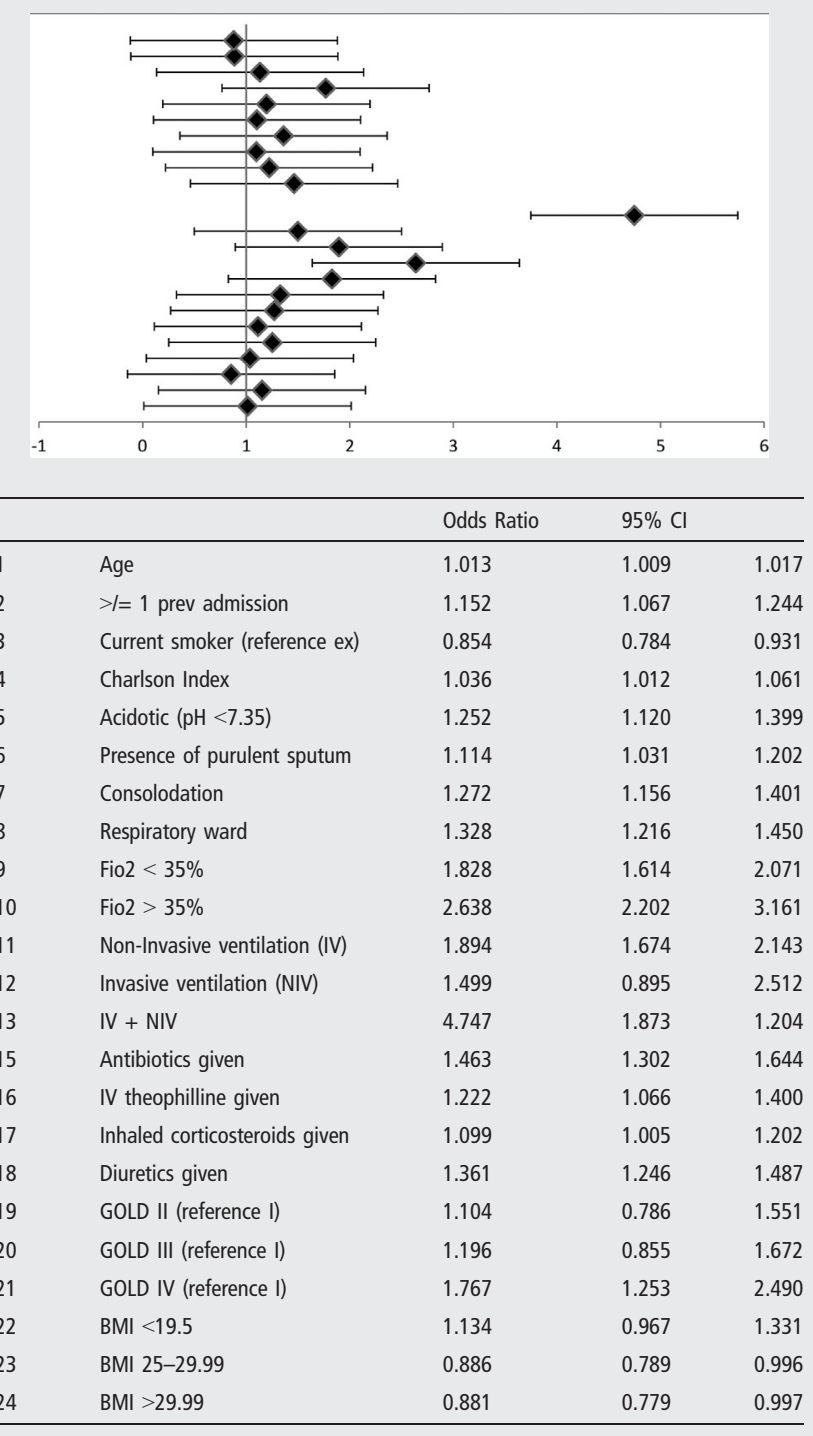

Introduction and objectives The major cost to health services of COPD care is hospital admission for exacerbation. Reducing length of stay (LOS) will reduce cost, yet there is wide variability across patients and hospitals. We test the hypothesis that these variations may be attributed to either patient characteristics, hospital characteristics and/or the so-called hospital-clustering effect, which indicates that patients with similar characteristics may experience different processes of care and outcomes depending on the hospital to which they are admitted.

Methods The European COPD Audit which was carried out in 432 hospitals from 13 countries, included data from 16,018 patients admitted over an 8 week period. The recorded variables included information on the patient and disease characteristics, resources available and clinical practice. Variables in each category associated with LOS were evaluated by a multivariate multilevel analysis and expressed as odds ratios (OR).

Results Mean LOS was 8.7 days (median: 7, standard deviation: 8.3, interquartile range: 4-11). Factors associated with an LOS higher than the median (see figure) were clinical with the highest impact in patients with use of mechanical ventilation (OR: 4.74) and higher oxygen flows (OR 2.63). In-hospital treatments, comorbidities and patient-related variables including GOLD class IV (OR 1.77) were also significant. These relationships were maintained with respect to longer LOS ( $>21$ days). Neither the day of admission, nor any of the resource variables were associated with significant differences in LOS. The crude variability of LOS between the different countries was reduced after accounting for these clinical factors and the clustering effect.

Conclusions This study demonstrates a noteworthy reduction in the observed crude inter-hospital variation in LOS after accounting for the hospital-cluster effect and patient related variables. This emphasises the predictor importance of the patients' clinical conditions and interventions, and understates the impacts of hospital resources and organisational factors. This "real-life" reflection may highlight some valid learning points that may help us to determine which achievable strategies are most relevant to improve outcomes.

\section{S107 EXPLAINING THE MORTALITY GAP IN COPD PATIENTS AFTER MYOCARDIAL INFARCTION: DATA FROM THE UK MYOCARDIAL ISCHAEMIA NATIONAL AUDIT PROJECT (MINAP)}

${ }^{1} \mathrm{KJ}$ Rothnie, ${ }^{1} \mathrm{~L}$ Smeeth, ${ }^{1} \mathrm{E}$ Herrett, ${ }^{1} \mathrm{~N}$ Pearce, ${ }^{2} \mathrm{H}$ Hemingway, ${ }^{3} \mathrm{~A}$ Timmis, ${ }^{4} \mathrm{~J}$ Wedzicha, ${ }^{1} \mathrm{JK}$ Quint. 'London School of Hygiene and Tropical Medicine, London, UK; ${ }^{2}$ University College London, London, UK; ${ }^{3}$ Barts and the London School of Medicine, London, UK; ${ }^{4}$ Imperial College London, London, UK

\subsection{6/thoraxjnl-2014-206260.113}

Introduction COPD patients are at increased risk of myocardial infarction (MI) and have increased mortality after an MI. Although some of this increased risk may be due to COPD itself, differences in management after an MI may play a role. ${ }^{1}$ We therefore investigated whether the increased in-hospital and 180 day mortality for COPD patients could be explained by differences in in-hospital and discharge treatment.

Methods Patients with a first MI between 2003-2013 were identified from the UK MINAP database. COPD patients had a record of obstructive airway disease, smoking history and were aged $>35$ years. Logistic regression was used to compare mortality in-hospital and at 180 days post-discharge between COPD and non-COPD patients. All models were adjusted for age, sex, smoking, previous cardiovascular disease, renal failure, diabetes and cardiovascular drugs used on admission. Variables relating to in-hospital management (delay in diagnosis, use of reperfusion and time to reperfusion/use of angiography in-hospital) and use

Abstract S107 Table 1 Differences in mortality after an MI between COPD and non-COPD patients. All odds ratios compare COPD to non-COPD patients

\begin{tabular}{llll}
\hline & $\begin{array}{l}\text { Minimally } \\
\text { adjusted OR } \\
(95 \% \mathrm{Cl})\end{array}$ & $\begin{array}{l}\text { Adjusted for in- } \\
\text { hospital factors } \\
\text { OR }(95 \% \mathrm{Cl})\end{array}$ & $\begin{array}{l}\text { Adjusted for use } \\
\text { of secondary } \\
\text { prevention } \\
\text { OR }(95 \% \mathrm{Cl})\end{array}$ \\
\hline $\begin{array}{l}\text { STEMIs } \\
\text { In-hospital mortality }\end{array}$ & $1.24(1.10-1.41)$ & $1.11(0.91-1.35)$ & - \\
$\begin{array}{l}180 \text { day mortality } \\
\text { Non-STEMls }\end{array}$ & $1.45(1.33-1.59)$ & $1.45(1.34-1.57)$ & $1.29(1.18-1.41)$ \\
$\begin{array}{l}\text { In-hospital mortality } \\
180 \text { day mortality }\end{array}$ & $1.34(1.24-1.45)$ & $1.16(1.07-1.26)$ & - \\
\hline
\end{tabular}


of secondary prevention on discharge were then sequentially added to models to assess the extent to which they explained the mortality difference.

Results 300,146 patients with a first MI were identified. 34,027 (11.3\%) had COPD. In-hospital mortality was greater for COPD patients after a STEMI (see Table 1), this difference was reduced after adjusting for in-hospital factors. Mortality was also greater for COPD patients at 180 days; this was not reduced after adjustment for in-hospital factors, but was reduced after adjusting for use of secondary prevention. In-hospital mortality was also greater for COPD patients after a non-STEMI, this was reduced after adjusting for in-hospital factors. Mortality at 180days after a non-STEMI was greater for COPD patients, this was reduced after adjusting for in-hospital factors, but not after adjusting for use of secondary prevention.

Conclusions Improved recognition and timely use of reperfusion treatments after a STEMI may significantly reduce the in-hospital mortality for COPD patients. Longer term mortality in COPD patients after a STEMI may be improved by increased use of secondary prevention drugs. Increased use of timely angiography may improve mortality for COPD patients after a non-STEMI.

\section{REFERENCE}

1 Quint, JK et al. BMJ. 2013;347:f6650

\section{Scientific advances in lung cancer}

\section{S108 MIF AS THE KEY REGULATOR FOR MESENCHYMAL STEM CELLS HOMING TO TUMOURS BY 3D AND IN VIVO LUNG METASTASIS MODELS}

${ }^{1} \mathrm{~S}$ Lourenco, ${ }^{1} \mathrm{VH}$ Teixeira, ${ }^{1} \mathrm{~T}$ Kalber, ${ }^{1} \mathrm{R}$ Thakrar, ${ }^{2} \mathrm{~A}$ Floto, ${ }^{1} \mathrm{SM}$ Janes. ${ }^{1}$ University College London, London, UK; ${ }^{2}$ Cambridge University, Cambridge, UK

10.1136/thoraxjn-2014-206260.114

Mesenchymal stromal cells (MSCs) are inherently tumour-homing and can be isolated, expanded and transduced, making them viable candidates for cell therapy. This tumour-tropism has been used to deliver anti-cancer therapies to various tumour models in several organs. In a previous study we have shown that MIF is the key director of MSC migration and infiltration towards tumour cells. We have shown this major role for MIF (mainly via CXCR4), using in vitro migration and invasion assays, in presence of different receptor inhibitors and achieving a drastic decrease in both processes using MIF inhibitor. Importantly we show that knock down of either CXCR4 or MIF abrogates MSC homing to tumours in an in vivo pulmonary metastasis model, confirming the in vitro $2 \mathrm{D}$ and $3 \mathrm{D}$ assays. In this study we define the mechanism behind MIF stimulation of MSC homing to tumours. We show that MIF upregulates other cytokines involved in chemotaxis, such as IL6, IL8 and CCL2 and upregulates MIF as well, amplifying the initial trigger and generating a positive feedback loop. However when inhibiting those cytokines individually, we never achieved a decrease in migration as drastic as for MIF inhibition. This suggests that the up-regulation of this set of cytokines would lead to chemoattraction of leucocytes to the site of the tumour, which was observed in a $3 \mathrm{D}$ model. Therefore, MIF trigger is amplified by its own upregulation in MSCs via a positive feedback loop, confirming again our previous findings and its key role as a regulator of MSC homing to tumours. This improved understanding of MSC tumour tropism will further enable development of novel cellular therapies for cancers.
$\$ 109$

MESENCHYMAL STEM CELLS EXPRESSING FULL LENGTH TRAIL - A PROMISING THERAPY FOR CANCER

ZQ Yuan, KK Kolluri, SM Janes. University College London, London, UK

\subsection{6/thoraxjnl-2014-206260.115}

Bone marrow derived mesenchymal stem cells (MSC) are promising tools for lung cancer therapy considering their tendencies for tumour homing and low immunogenicity. Tumour necrosis factor related apoptosis inducing ligand (TRAIL) is a pro-apoptotic protein that induces selective apoptosis of tumour cells, while sparing normal cells. Therefore, it is expected that MSCs engineered to produce TRAIL will home to and kill cancer cells.

In this study, two lentiviral vectors were constructed to express the full-length (flT) or a truncated soluble form of TRAIL (sT) driven by a CMV promoter/enhancer. A secretion targeting sequence and an isoleucine zipper (ILZ) peptide were sequentially added to the $\mathrm{N}$-terminal of the soluble TRAIL to produce secreted and trimerised TRAIL. TRAIL lentiviruses were prepared and human BM-MSCs were transduced with a multiplicity of infection (MOI) of 2. FACS analysis by anti-TRAIL antibody staining demonstrated that over $99 \%$ of flT or sT viruses transduced cells are positive for TRAIL expression. TRAIL expression was further confirmed by Western blotting and ELISA assays. The flT or sT expressing MSCs both showed similar level of cellular TRAIL expression ( 350 ng TRAIL per $1 \mathrm{mg}$ of total cellular protein).

Co-culture of cancer cells with transduced MSCs determined the cancer killing efficacy of MSCs expressing flT or sT. Twenty cancer cell lines were tested and classified into four TRAIL response groups; high, medium, low, and no sensitivity to recombinant TRAIL (rTRAIL) at the concentration of $50 \mathrm{ng} / \mathrm{ml}$. At the co-culture ratio of 4:1 cancer to MSC cells, MSC-sT treatment showed no or only marginal cancer cell killing effect, in contrast, MSC-flT showed promising effects on all tested cell lines, with an apoptosis induction rate ranging between $35-75 \%$. In groups designated as high, moderate and low, MSC-flT are as effective as rTRAIL and induced marked cell death $(\mathrm{p}<0.001)$ in cell lines which showed no sensitivity to rTRAIL (Figure).

In conclusion, these results demonstrate MSC-flT is a promising cell therapy and have great potential for clinical treatment of lung cancers and pleural metastases.

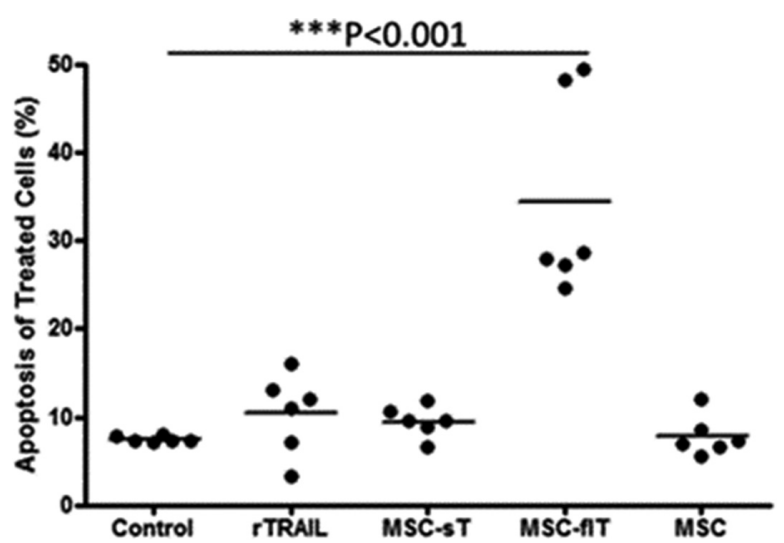

Abstract S109 Figure 1 MSC-fIT cells induce apoptosis in recombinant TRAIL-resistant cancer cells. Six rTRAlL-resistant cancer cell lines were treated with $50 \mathrm{ng} / \mathrm{ml}$ rTRAIL, MSC, MSC-sT and MSC-fIT cells for $24 \mathrm{~h}$. Apoptosis was quantified by Annexin VIDAPIFACS 